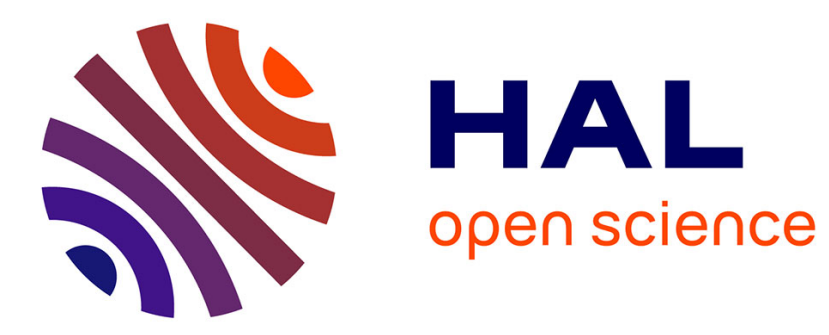

\title{
Un homme de directives
}

Christian Gauberville

\section{- To cite this version:}

Christian Gauberville. Un homme de directives. Revue forestière française, 2006, 58 (2), pp.109-113. 10.4267/2042/5808 . hal-03449398

\section{HAL Id: hal-03449398 https://hal.science/hal-03449398}

Submitted on 25 Nov 2021

HAL is a multi-disciplinary open access archive for the deposit and dissemination of scientific research documents, whether they are published or not. The documents may come from teaching and research institutions in France or abroad, or from public or private research centers.
L'archive ouverte pluridisciplinaire HAL, est destinée au dépôt et à la diffusion de documents scientifiques de niveau recherche, publiés ou non, émanant des établissements d'enseignement et de recherche français ou étrangers, des laboratoires publics ou privés. 


\section{UN HOMME DE DIRECTIVES}

\section{Christian Gauberville}

Le 21 mai 1992, la directive 92/43/CEE du Conseil concernant la conservation des habitats naturels ainsi que de la faune et de la flore sauvages dite directive habitats paraissait au Journal officiel de la Communauté européenne.

Jean-Claude Rameau, comme un certain nombre d'autres scientifiques, savait que ce texte était en gestation mais n'avait pas été directement impliqué dans sa genèse. Néanmoins, par deux aspects au moins, le texte et ses implications exerçaient sur lui une forte attirance voire une nécessité absolue d'agir : le concept même d'habitat, très intégrateur, quasi synonyme d'écosystème pour les écologues français et à ce titre incluant dans sa définition la faune, et le volet dynamique de végétation aboutissant en Europe tempérée, sauf rares exceptions, à un groupement végétal dominé par la forêt. Le phytosociologue devenu phytoécologue avait là une opportunité de nourrir son esprit de synthèse et d'entreprendre les actions lui permettant d'atteindre son objectif : aider le gestionnaire forestier, selon lui clé de voûte et garant d'une gestion durable de ces milieux et du maintien de la biodiversité qu'ils hébergent.

Il y voit la possibilité de faire rentrer dans le concret le concept de gestion intégrée des forêts, c'est-à-dire la prise en compte consciente des questions de biodiversité dans les actes quotidiens de gestion, d'en expliquer le bien-fondé et d'initier des processus de sensibilisation : c'est la Convention de Rio dans les aménagements forestiers et les plans simples de gestion... mais librement consentie car bien expliquée et bien comprise.

\section{VOUS AVEZ DIT “ DIRECTIVE ” ?}

Très vite, le dossier prend de l'ampleur et de multiples tâches lui apparaissent indispensables : d'abord rendre lisibles les documents techniques officiels, traduire au propre et au figure Corine biotopes, EUR 12, puis EUR 15, le Manuel officiel d'interprétation des habitats d'intérêt européen, ensuite construire des outils pour le gestionnaire dans différents domaines comme la détermination des habitats, leur cartographie à l'échelle de la gestion, l'évaluation de leur état de conservation et enfin vulgariser, en commençant par le commencement pour un professeur, c'est-à-dire appliquer tout cela dans son enseignement.

Sollicité pour faire partie du groupe d'experts français chargé d'appuyer la démarche, il entreprend tout d'abord la refonte de certains codes de Corine biotopes, document de base de la classification des habitats européens mais souvent trop vaste pour être efficace au niveau d'un pays, d'autant que sont fréquemment mélangées références phytosociologiques et références physionomiques. Toujours avec le souci de faciliter le travail aux hommes de terrain, le spécialiste de la dynamique forestière pointera quelques incohérences du document pour en rendre son application plus facile ; c'est ainsi que seront intégrés les codes des saulaies, groupements pionniers conduisant à des forêts plus mûres qui, elles, étaient visées par la directive ${ }^{(1)}$. Il était en effet inenvisageable de prendre en compte l'un sans l'autre en particulier pour cartographier

(1) Rappelons que, dans le texte initial de la directive, les habitats de l'annexe 1 étaient désignés par leur code Corine ; ce n'est que dans la version modifiée de 1997 que sont apparus les codes Natura 2000. 
leurs mosaïques... De la même manière, les faciès de reconquête à Frênes, Ormes, Érables ou Tilleuls, forêts secondaires de nombreuses régions françaises suite aux différentes vagues de déprise agricole, non concernées par la directive, seront bien distingués des groupements de ravins sur éboulis, forêts mûres liées à des blocages stationnels et fortement originales par rapport aux types de forêts régionales environnantes.

C'est ainsi l'ensemble des codes Corine qui sont revisités, validés ou amendés, avec le double objectif d'être utile au praticien et de garder la cohérence avec le système phytosociologique français qui, dans le même temps, est en voie de structuration : l'exercice en soi, comme les discussions avec ses collègues experts, seront forcément ardus.

De nombreuses précisions ont été apportées mais toutes les propositions n'ont pas été retenues ; c'est ainsi qu'il lui apparaissait absurde d'intégrer les hêtraies neutrophiles montagnardes dans le réseau au titre des hêtraies à Aspérule, non pas parce qu'elles relèveraient d'une autre unité phytosociologique mais parce que ces groupements concernent des surfaces considérables dans le Nord-Est et l'Est du pays ; pour lui, l'esprit des textes était dès lors largement transgressé. Sur ce point, il n'eut pas gain de cause.

À partir de 1993, le travail se structure, les relations avec les experts français et ceux de Bruxelles se font plus fréquentes. Au niveau de la Commission, il s'agit de finaliser le Manuel d'interprétation des habitats de l'Union et Jean-Claude Rameau y apporte sa part en même temps qu'il travaille à la déclinaison française, en particulier en tant que président de la zone biogéographique continentale.

C'est en 1993 qu'il rédigera, édité sous le timbre du Muséum national d'Histoire naturelle, un manuel détaillé des habitats forestiers ; ce document précise les groupements concernés par la directive, en donne les caractéristiques majeures nécessaires à leur identification, reprenant pour cela les travaux antérieurs de mise en cohérence de la typologie Corine. Il précise leur variabilité et propose quelques pistes générales pour leur gestion.

Puis en 1994 et 1995, les travaux et les réunions se succèdent en France, en Allemagne (Bonn, domaine continental), en Belgique (Bruxelles), en Italie (Rome, domaine alpin)...

L'année 1996 constitue probablement une période charnière en ce sens que plusieurs grands programmes liés à la directive habitats l'ont mis à contribution, auxquels il faut ajouter un événement politique qui l'a probablement assez affecté. À cette date, l'IDF venait d'obtenir un programme LIFE où l'ONF et l'ENGREF, mais pour cette dernière, plus précisément le "Laboratoire d'étude des écosystèmes forestiers et de la dynamique des paysages" était directement impliqué ; il s'agissait de mettre en place des outils de transfert de connaissance des habitats vers les propriétaires et les gestionnaires. Le travail envisagé était important, concernait trois pays de l'Union européenne (la France, la Wallonie pour la Belgique et le Grand-Duché de Luxembourg), et était prévu sur une durée de trois ans. Jean-Claude Rameau assura la rédaction de la partie scientifique et descriptive de l'ensemble des habitats élémentaires concernés. Dans le courant de cette même année, l'ONF le sollicitera pour un travail considérable : évaluer et hiérarchiser les propositions de sites pour les forêts publiques.

\section{AU CEUR DE L'ACTION !}

C'est à cette époque que le monde rural, contestant la mise en œuvre du réseau Natura 2000 plus sur la forme que sur le fond, déclenche une vague de froid qui entraîne un gel gouverne- 
mental (tardif ou précoce, c'est selon !) du processus de mise en place de la directive en plein mois de juillet (le 19).

Jean-Claude Rameau y voit là une négation des efforts consentis par les scientifiques et les vulgarisateurs (il peut se réclamer des deux) et tente, de façon positive, d'en tirer les leçons dans les colonnes de cette même revue en 1997. Dans un article de 16 pages "La directive habitats : analyse d'un échec, réflexions pour l'avenir", il détaille "le grand intérêt de la philosophie de la directive " et "ses objectifs excellents" (la gestion intégrée des habitats pour le maintien de la biodiversité), il déplore "la confusion entre information et concertation » ayant mené à " une cristallisation des réactions hostiles à Natura 2000 ", montre que malgré tout de nombreux travaux d'intérêt pour l'avenir sont mis en œuvre parallèlement au " déroulement chaotique $d u$ dossier " et, enfin, dégage les principes fondamentaux d'une réelle concertation apte à dégager un vrai consensus.

Son implication était alors totale et ne faiblira pas. La relance de Natura 2000 a lieu, lui ouvrant de nouvelles possibilités d'intervention ; sur la demande pressante des acteurs du monde rural, les ministères de l'Environnement et de l'Agriculture dégagent les moyens d'une mise en œuvre des cahiers d'habitats. On commença par les milieux forestiers et, après quelques tâtonnements, scientifiques et gestionnaires se retrouvaient dans le même groupe de travail. Jean-Claude Rameau rédigeait les premiers textes qui étaient discutés en séance sous la houlette modératrice de spécialistes de la gestion des conflits. Durant cette époque formidable, il fallut plusieurs mois pour mettre au point le cahier "modèle" de la hêtraie à Luzule blanchâtre ; pour calmer son impatience, Jean-Claude Rameau pestait, interpellait les uns et bousculait les autres sans distinction d’âge ou de grade et "modérait” à la place des modérateurs.

En 1996 enfin, il termine la compilation des habitats forestiers français à partir de l'analyse de la bibliographie phytosociologique disponible ; il y fait part dans un tome d'introduction de ses conceptions pour le synsystème français dont il avait déjà posé les bases dans sa thèse de doctorat d’État parue en 1987.

Les trois guides LIFE (France domaines atlantique et continental, et Wallonie - Grand-Duché de Luxembourg), les deux tomes des cahiers d'habitat forestier, deux œuvres collectives auxquelles il apporta une contribution majeure auront été, à côté des outils pratiques qu'il appelait de ses vœux pour les gestionnaires forestiers, l'opportunité de montrer les liens existant entre phytosociologie, station et habitat.

"Ce qui est étonnant chez Rameau c'est qu’il détermine les stations en avançant dans les parcelles » me disait à la fin des années 1980 un directeur de CRPF ; en fait, Jean-Claude Rameau gardait en mémoire la structuration des grandes unités de végétation et les déclinait régionalement en fonction des particularités climatiques et géologiques ; à cette époque-là, déjà, les stations étaient recalées par rapport aux grands types d'habitat.

Ces habitats mis sur le devant de la scène par une directive européenne seront désormais la base de ses travaux.

Ayant dans un premier temps approfondi et structuré les connaissances dans le domaine continental, il étudia la répartition générale des habitats forestiers au niveau du territoire français de même que leur dynamique.

Les habitats associés recelant fréquemment des espèces rares ou particulières, bien souvent plus que la forêt elle-même, Jean-Claude Rameau ne manquait jamais de les intégrer dans toute étude, considérant que le gestionnaire devait être informé de la biodiversité existant dans les espaces dont il avait la charge. 
Il mit en route une quantité considérable de travaux dans divers cadres de son enseignement : élèves de la FIF, étudiants du Mastère, élèves-ingénieurs du GREF, thésards ont tous travaillé à appréhender les habitats, les déterminer, les cartographier, en préciser leur dynamique, rédiger des clés pour leur détermination et in fine travailler à l'élaboration de mesures de gestion crédibles pour le gestionnaire.

L'année dernière, il prenait la tête d'un groupe de travail piloté par le Muséum national d'Histoire naturelle pour travailler sur l'état de conservation des habitats forestiers dans le cadre de l'évaluation de la contribution du réseau Natura 2000 au maintien de la biodiversité. Non satisfait du degré de connaissance actuel de la répartition des habitats forestiers au niveau de l'ensemble du territoire et travaillant pour l'avenir, il faisait dès le début des travaux des propositions concrètes pour améliorer rapidement la situation.

\section{LES HABITATS, SUJETS D’ÉTUDE ET D’ÉMERVEILLEMENT}

Les demandes d'expertises l'entraînaient à parcourir la France de telle façon que les points cardinaux n'avaient plus vraiment de sens pour son fidèle véhicule de l'ENGREF de Nancy... Ayant longtemps écumé les hêtraies sèches, les marais alcalins à Swertia perennis, les hêtraies à Aspérule, les tufières géantes et les frênaies-érablaies alluviales calcicoles de sa chère HauteMarne, il a porté ensuite une vraie admiration pour les paysages et les divers habitats forestiers et associés de Bourgogne, du Jura et surtout des Alpes. Avec ses élèves, plusieurs vallées de cette vaste région furent étudiées mais deux sites l'inspiraient plus particulièrement : le bassin de Gap-Chaudin où il mit en œuvre une activité de recherche sur l'impact des modes de gestion forestière sur la biodiversité dans le contexte d'un bassin versant, avec des forêts représentatives des Alpes du Sud, et le Mercantour avec la Vallée des merveilles, les Pineraies à crochets, les formations à Pinus mugo, les landines à Azalée naine et d'autres habitats encore qu'il faisait découvrir aux étudiants et où, trop tôt, son histoire s'est terminée.

Les habitats forestiers et associés ont été pour lui des sujets d'étude mais également des sujets d'émerveillement ; deux habitats particulièrement originaux pour la France avaient plus que tout autre ses faveurs, les formations de Genévriers thurifères en particulier les arbres vénérables de Saint-Crépin dans les Hautes-Alpes et les étonnants Pins à crochets ou Épicéas nains sur sol gelé du Subalpin que l'on trouve ici ou là à l'abri de falaises froides dans les Alpes et le Jura.

Mais la curiosité toujours renouvelée de l'écologue pour les "sciences naturelles" n'était qu'un aspect des centres d'intérêts multiples de cet homme cultivé et, évoquant la visite des Pins de Salzmann accrochés à la colline de Saint-Guilhem-le-Désert, il gardait le souvenir ému de la beauté dégagée par l'unité architecturale du village.

Passionné par la forêt sous toutes ses formes, expert des multiples voies de la dynamique des végétations forestières, Jean-Claude Rameau a très tôt compris l'intérêt du concept d'habitat dans les applications de gestion quotidienne, comme dans la mise en place de travaux plus fondamentaux liés au suivi scientifique de réserve biologique par exemple.

Sachant concilier recherche et vulgarisation de ses résultats, doté d'un esprit de synthèse hors du commun, il parcourait la France en tout sens pour répondre aux sollicitations d'expertises toujours plus nombreuses en même temps qu'il assurait son enseignement en y développant de nouvelles approches et qu'il organisait des stages de formation continue.

Aider le gestionnaire encore et encore ! 
La quantité impressionnante de travaux qu'il a effectués seul, comme avec ses élèves, allait bientôt nécessiter une grande synthèse ; elle mûrissait lentement dans son esprit... pour la retraite.

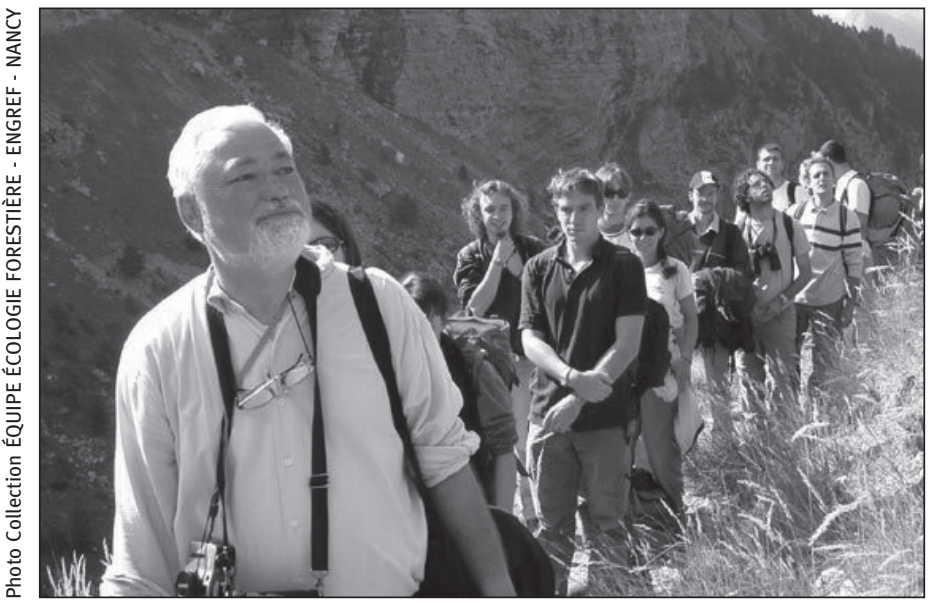

